\title{
CONTROL END-EFFECTOR MOVEMENT OF ROBOTIC ARM BY PSEUDOINVERSE METHOD
}

\author{
Ondrej HOCK, Jozef SEDO \\ Department of Mechatronics and Electronics, Faculty of Electrical Engineering, \\ University of Žilina, Univerzitná 8215/1, 01026 Žilina, Slovakia, \{ondrej.hock, jozef.sedo\}@fel.uniza.sk
}

\begin{abstract}
Inverse kinematics and pseudoinverse method for controlling movement of robotic arm is presented in this paper. For that task we used robotic arm DOBOT for which we made simulation model in Matlab environment and SimMechanics toolbox. We described in detail pseudoinverse method and problem solving of pseudoinverse for non-square matrixes. Because in future we will make implementation this method to movement control of robotic arm, we made simulation, how many digits we will need for correctly calculating of goniometrics functions in DSP.
\end{abstract}

Keywords: Inverse kinematics, Matlab Simulink simulation, robotic arm, pseudoinverse method, SimMechanics.

\section{INTRODUCTION}

Complex control systems nowadays are designed for wide range of industrial applications. They are focused on effective management of composite processes, energy savings and optimization of production and technological processes. For this reason industry is using robotic manipulators for which we need to know the end-effector kinematics [1].

The robot kinematics can be divided into forward kinematics and inverse kinematics. Forward kinematics problem is straightforward and there is no complexity deriving the equations [2]. Hence, there is always a forward kinematics solution of a manipulator. Inverse kinematics is a much more difficult problem than forward kinematics. The solution of the inverse kinematics problem is computationally expansive and generally takes a very long time in the real time control of manipulators. Singularities and nonlinearities that make the problem more difficult to solve. Hence, only for a very small class of kinematically simple manipulators (manipulators with Euler wrist) have complete analytical solutions [3-5].

Two main solution techniques for the inverse kinematics problem are analytical and numerical methods. In the first type, the joint variables are solved analytically according to given configuration data. In the second type of solution, the joint variables are obtained based on the numerical techniques. In this chapter, the analytical solution of the manipulators is examined rather than numerical solution [6-8].

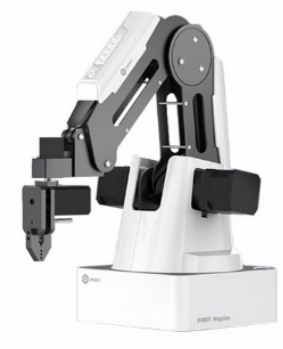

Fig. 1 DOBOT Magician [8]
The whole paper will be dedicated to the robot arm DOBOT Magician (hereafter DOBOT) shown in Figure 1.

\section{THEORY OF PSEUDOINVERSE METHOD}

The pseudoinverse method sets the value $\Delta \theta$ equal to

$\Delta \theta=J^{\dagger} \vec{e}$

where the $n \times m$ matrix $J^{\dagger}$ is the pseudoinverse of $J$, also called the Moore-Penrose inverse of $J$. It is defined for all matrices $J$, even ones which are not square or not of full row rank. The pseudoinverse gives the best possible solution to the equation $J \Delta \theta=\vec{e}$ in the sense of least squares. In particular, the pseudoinverse has the following nice properties. Let $\Delta \theta$ be defined by equation (1) [9]. First, suppose $\vec{e}$ is in the range (i.e., the column span) of $J$. In this case, $J \Delta \theta=\vec{e}$; furthermore, $\Delta \theta$ is the unique vector of smallest magnitude satisfying $J \Delta \theta=\vec{e}$. Second, suppose that $\vec{e}$ is not in the range of $J$. In this case, $J \Delta \theta=\vec{e}$ is impossible. However, $\Delta \theta$ has the property that it minimizes the magnitude of the difference $J \Delta \theta-\vec{e}$. Furthermore, $\Delta \theta$ is the unique vector of smallest magnitude which minimizes $\|J \Delta \theta-\vec{e}\|$, or equivalently, which minimizes $\|J \Delta \theta-\vec{e}\|^{2}[9,10]$.

The pseudoinverse tends to have stability problems in the neighbourhoods of singularities. At a singularity, the Jacobian matrix no longer has full row rank, corresponding to the fact that there is a direction of movement of the end effectors which is not achievable [11,12]. If the configuration is exactly at a singularity, then the pseudoinverse method will not attempt to move in an impossible direction, and the pseudoinverse will be wellbehaved. However, if the configuration is close to a singularity, then the pseudoinverse method will lead to very large changes in joint angles, even for small movements in the target position. In practice, roundoff errors mean that true singularities are rarely reached and instead singularity have to be detected by checking values for being near-zero $[13,14]$.

The pseudoinverse has the further property that the matrix $\left(I-J^{\dagger} J\right)$ performs a projection onto the nullspace 
of $J$. Therefore, for all vectors $\varphi, J\left(I-J^{\dagger} J\right) \varphi=0$. This $J=U \cdot \Sigma \cdot V^{T}$

means that we can set $\Delta \theta$ by

$\Delta \theta=J^{\dagger} \vec{e}+\left(I-J^{\dagger} J\right) \varphi$

for any vector $\varphi$ and still obtain a value for $\Delta \theta$ which minimizes the value $J \Delta \theta-\vec{e}$. By suitably choosing $\varphi$, one can try to achieve secondary goals in addition to having the end effectors track the target positions. For instance, $\varphi$ might be chosen to try to return the joint angles back to rest positions,: this can help avoid singular configurations $[9,10]$.

An algorithm for the pseudoinverse method can be derived as follows:

From equation (5), we get the normal equation

$J^{T} J \Delta \theta=J^{T} \vec{e}$

Then we let $\vec{z}=J^{T} \vec{e}$ and solve the equation

$\left(J^{T} J\right) \Delta \theta=\vec{z}$

Now it can be shown that $\vec{z}$ is always in the range of $J^{T} J$, hence equation (4) always has a solution. In principle, row operations can be used to find the solution to (4) with minimum magnitude; however, in the neighborhood of singularities, the algorithm is inherently numerically unstable $[9,15]$.

When $J$ has full row rank, then $J J^{T}$ is guaranteed to be invertible. In this case, the minimum magnitude solution $\Delta \theta$ to equation (4) can be expressed as

$\Delta \theta=J^{T}\left(J J^{T}\right)^{-1} \vec{e}$

To prove this, note that if $\Delta \theta$ satisfies (5), then $\Delta \theta$ is in the row span of $J$ and $\Delta \theta=\overrightarrow{\mathrm{e}}$. Equation (5) cannot be used if $J$ does not have full row rank. A general formula for the pseudoinverse for $J$ not of full row rank can be found in [9,12].

The pseudoinverse method is widely discussed in the literature but it often performs poorly because of instability near singularities [16].

\section{PROBLEM SOLVING OF PSEUDOINVERSE METHOD}

In case, the number of independent coordinates (rotation of individual joints) is greater than the number of coordinates of the manipulator endpoint (in space 6, for translational motion 3 and 3 for rotation), the redundancy problem will arise. In this case, there can generally be infinitely many combinations of independent co-ordinates for one endpoint positioning. The Jacobi matrix has the dimensions of $m$ lines and $n$ columns $(m \neq n)$, ie. $J$ is the non-square matrix. Generally, it is not possible to calculate the inverse matrix from the non-matrix matrix.

In order to address the inverse role of kinematics in such cases, pseudo-inversion of the Jacobi matrix (designation $\mathrm{J}^{+}$). This method uses SVD (Singular value decomposition) of the Jacobi matrix for determination $\mathrm{J}^{+}$.

We can decompose every matrix $J$, which is Jacobi matrix, by SVD decompose for 3 matrixes:
Where:

- $\mathrm{J}$ is Jacobi matrix is matrix with dimension mxn

- $\mathrm{U}$ is ortogonal matrix with dimension mxm, ie. $\mathrm{U}^{-1}=\mathrm{U}^{\mathrm{T}}$

- $\quad \mathrm{V}$ is ortogonal matrix with dimension nxn, ie. $\mathrm{V}^{-1}=\mathrm{U}^{\mathrm{T}}$

- $\sum$ is diagonal matrix with dimension mxn, which on main diagonal contained singular numbers of matrix A

$\left[\begin{array}{cccc}j_{11} & j_{12} & \cdots & j_{1 n} \\ j_{21} & j_{22} & \cdots & j_{2 n} \\ \vdots & \vdots & \ddots & \vdots \\ j_{m 1} & j_{m 2} & \cdots & j_{m n}\end{array}\right]=\left[\begin{array}{cccc}u_{11} & u_{12} & \cdots & u_{1 m} \\ u_{21} & u_{22} & \cdots & u_{2 m} \\ \vdots & \vdots & \ddots & \vdots \\ u_{m 1} & u_{m 2} & \cdots & u_{m m}\end{array}\right]$
$\left[\begin{array}{cccc}\sigma_{1} & 0 & \cdots & 0 \\ 0 & \sigma_{2} & \cdots & 0 \\ \vdots & \vdots & \ddots & \vdots \\ 0 & 0 & \cdots & \sigma_{d}\end{array}\right] \cdot\left[\begin{array}{cccc}v_{11} & v_{21} & \cdots & v_{n 1} \\ v_{12} & v_{22} & \cdots & v_{n 2} \\ \vdots & \vdots & \ddots & \vdots \\ v_{1 n} & v_{2 n} & \cdots & v_{n n}\end{array}\right]$

Where: $d=m$ for $m<n$ and $d=n$ for $m>n$.

$$
\begin{aligned}
& J \cdot J^{T}=\left(U \cdot \Sigma \cdot V^{T}\right) \cdot\left(U \cdot \Sigma \cdot V^{T}\right)^{T} \\
& J \cdot J^{T}=U \cdot \Sigma \cdot V^{T} \cdot V \cdot \Sigma^{T} \cdot U^{T}
\end{aligned}
$$

$J \cdot J^{T}=U \cdot \Sigma \cdot I \cdot \Sigma^{T} \cdot U^{T}$

$J \cdot J^{T}=U \cdot \Sigma \cdot \Sigma^{T} \cdot U^{T}$

We multiply the equation 8 by $\mathrm{U}$ matrix for right:

$J J^{T} \cdot U=U \cdot \Sigma \Sigma^{T} \cdot U^{T} \cdot U$

$J J^{T} \cdot U=U \cdot \Sigma \Sigma^{T} . I$

$J J^{T} \cdot U=U \cdot \Sigma \Sigma^{T}$

We get problem of eigenvalues for matrix $J J^{T}$, where $U$ is square matrix of eigenvectors for matrix $J J^{T}$ and $\Sigma \Sigma^{T}$ is diagonal square matrix of eigenvalues $\lambda_{1}, \ldots, \lambda_{m}$.

$\Sigma . \Sigma^{T}=\left[\begin{array}{cccc}\sigma_{1} & 0 & \cdots & 0 \\ 0 & \sigma_{2} & \cdots & 0 \\ \vdots & \vdots & \ddots & \vdots \\ 0 & 0 & \cdots & \sigma_{d}\end{array}\right] \cdot\left[\begin{array}{cccc}\sigma_{1} & 0 & \cdots & 0 \\ 0 & \sigma_{2} & \cdots & 0 \\ \vdots & \vdots & \ddots & \vdots \\ 0 & 0 & \cdots & \sigma_{d}\end{array}\right]=$
$\left[\begin{array}{ccccc}\sigma_{1}^{2} & 0 & \cdots & 0 \\ 0 & \sigma_{2}^{2} & \cdots & 0 \\ \vdots & \vdots & \ddots & \vdots \\ 0 & 0 & \cdots & \sigma_{d}^{2}\end{array}\right]$

$J^{T} \cdot J=\left(U \cdot \Sigma \cdot V^{T}\right)^{T} \cdot\left(U \cdot \Sigma \cdot V^{T}\right)$

$J^{T} \cdot J=V \cdot \Sigma^{T} \cdot U^{T} \cdot U \cdot \Sigma \cdot V^{T}$

$J^{T} . J=V \cdot \Sigma^{T} \cdot I \cdot \Sigma \cdot V^{T}$

$J^{T} \cdot J=V \cdot \Sigma^{T} \cdot \Sigma \cdot V^{T}$ 
We multiply the equation 11 by $\mathrm{V}$ matrix for right:

$J^{T} J \cdot V=V \cdot \Sigma^{T} \Sigma \cdot V^{T} \cdot V$

$J^{T} J \cdot V=V \cdot \Sigma^{T} \Sigma . I$

$J^{T} J \cdot V=V \cdot \Sigma^{T} \Sigma$

We get the problem of eigenvalues for matrix $J^{T} J$, where $V$ is square matrix of eigenvectors for matrix $J^{T} J$ and $\sum^{T} \sum$ is diagonal square matrix of eigenvalues $\lambda_{1}, \ldots, \lambda_{n}$.

Matrices $J J^{T}$ and $J^{T} J$ are symmetric matrix and they have same non-zero eigenvalues. Eigenvalues of symmetric matrices are always the real values. Therefor we won't calculate eigenvalues of $J J^{T}$ again. Is necessary calculate only eigenvectors of $J J^{T}$.

\section{SIMULATION RESUTLS}

We made simulation for robotic arm in Matlab Simulink and also in m-file. Main idea was prepare for later implementation of pseudoinverse method to DSP. For that we made simulation for number of digit after comma and number of steps we necessarily needs. Simulation results of pseudoinverse method can be found in figures 2 to 4 and simulation results of necessarily number of digits and number of steps shown at figures 5 and 6 .

Figure 2 show end-effector movement of robotic arm. We design simulation for 3 reference coordinates as we can see in figure 2 . For $(50,90,80)$ is blue line, $(100,150,160)$ is red line and third coordinates was $(150,180,140)$ which is green line.

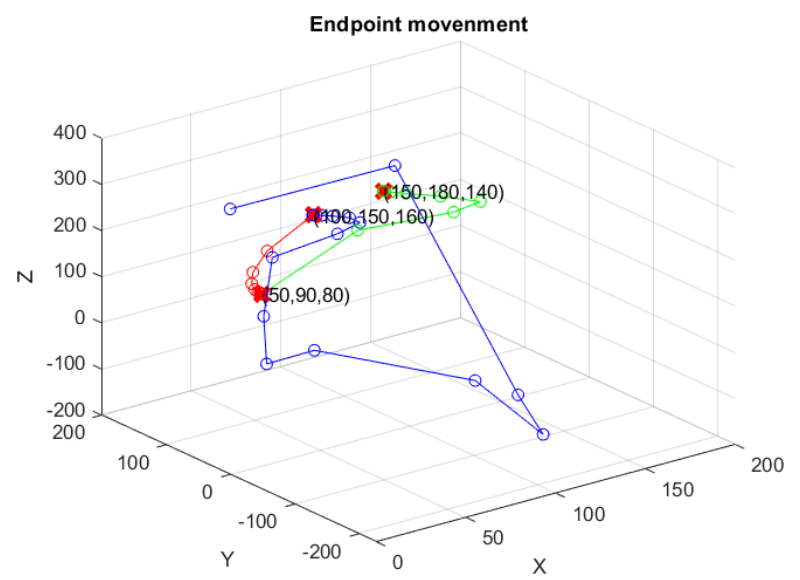

Fig. 2 Simulation result of pseudoinverse method for DOBOT manipulator

Figures 3 and 4 shows axis $\mathrm{X}, \mathrm{Y}$ and $\mathrm{Z}$ of robotic arm end-effector and total error of coordinates. Also figures show number of necessarily steps for each simulation. In first result we ask for maximal error of coordinates equal to $1 \mathrm{e}-3 \mathrm{~mm}$ and number of digits equal to 6 . In second figure we ask for maximal error equal to $1 \mathrm{e}-2 \mathrm{~mm}$ and number of digits equal to 5 .
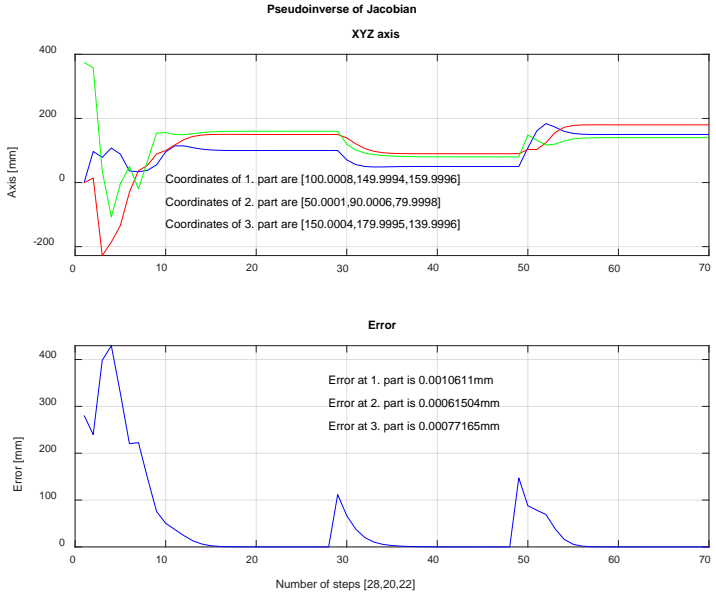

Fig. 3 Simulation result of DOBOT axis and total error of coordinates
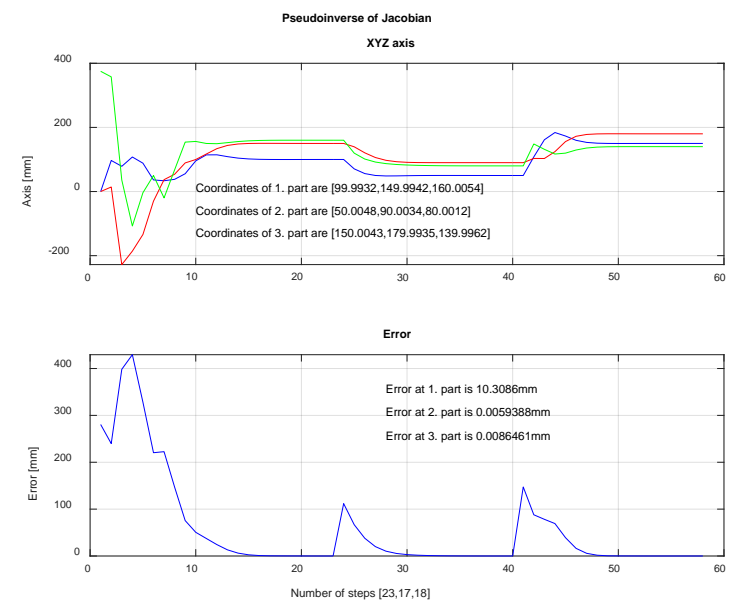

Fig. 4 Simulation result of DOBOT axis and total error of coordinates.

Since these simulations are a preparation for implementing a method into the DSP, is necessary know how many digits after point we needed. Number of digits and middle value of error is in Table 1 . Figure 5 show these table for each axis.

Table 1 Number of digits and middle value of error

$\begin{array}{cc}\text { Digits } & \text { Middle value of error [mm] } \\ 1 & 22,31 \\ 2 & 7,627 \\ 3 & 0,786 \\ 4 & 0,104 \\ 5 & 11,3 \mathrm{e}-4 \\ 6 & 8,3 \mathrm{e}-4\end{array}$




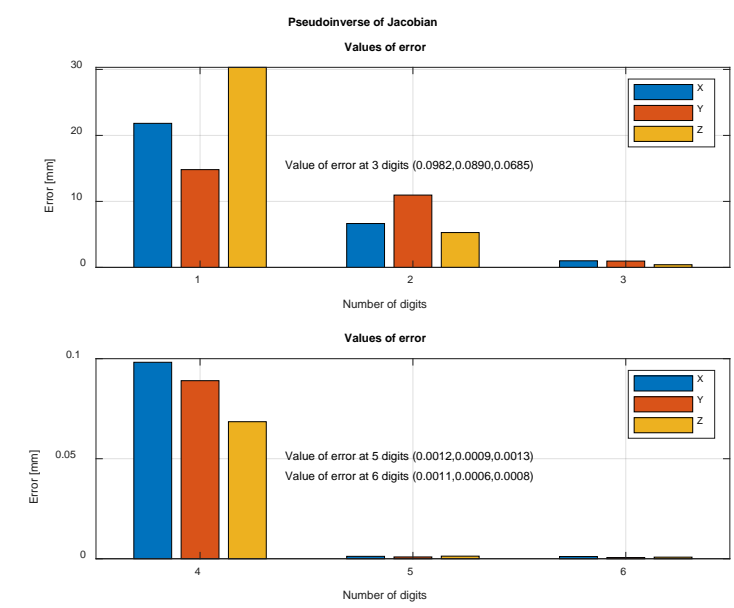

Fig. 5 Simulation result of total error of coordinates from 1 to 6 digit

In the Table 2 are numbers of simulation steps for reference error and for each reference coordinates from figure 2. Figure 6 graphically show the previous table.

Table 2 Number of simulation steps for different

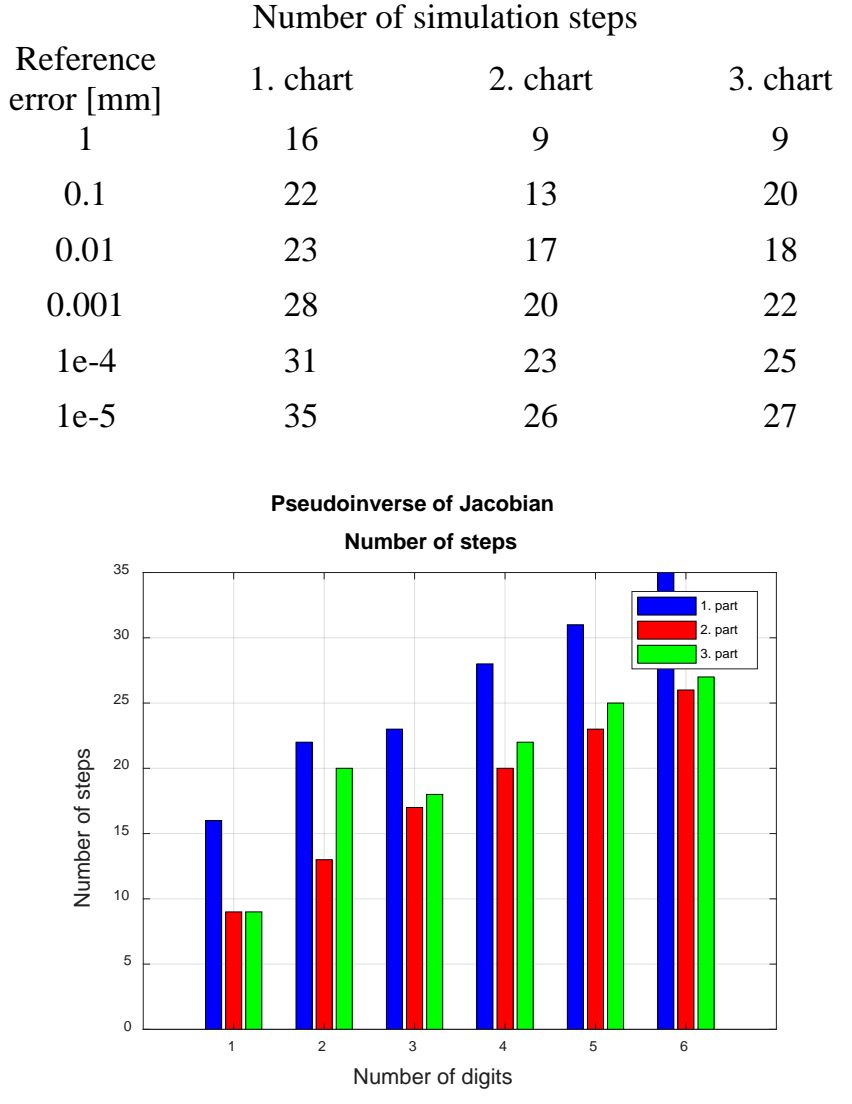

Fig. 6 Simulation result of total error of coordinates from 1 to 6 digit

\section{CONCLUSIONS}

In this paper we theoretically described pseudoinverse method for control end-effector movement of DOBOT manipulator. We needn't research rotation of end- effector because DOBOT has mechanically secures the retention of the end-effector in the plane and pad.

In near future we will test several implementation method of pseudoinverse to DSP. For reducing calculation time in DSP it is important to rightly implement the pseudoinverse method.

We have to use numbers with four decimal places at least, for the achievement of required precision $0.1 \mathrm{~mm}$. We can assume 20 iteration steps of the pseudoinverse method for the movement from place $A$ to place $B$ to achieve a structural precision of the DOBOT manipulator, i.e. 0.4 $\mathrm{mm}$.

For communication between DSP and DOBOT can be used USB, Wifi UDP, BLE and UART interface.

\section{ACKNOWLEDGMENTS}

The authors would like to thank to project KEGA 027ŽU-4/2018 Modelling, Design and Implementation of the Modern Method in the Educational Process of the Technical Faculties Focusing on Discrete Control of Power Systems.

\section{REFERENCES}

[1] VIRGALA, I. - KELEMEN, M. - PRADA, E.: et al.: Motion analysis of snake robot segment, In: 11th IEEE International Symposium on Applied Machine Intelligence and Informatics (SAMI), Pages: 145-148 Published: 2013.

[2] CHOVANCOVA, A. - FICO, T. - HUBINSKY, P.: et al.: Comparison of various quaternion-based control methods applied to quadrotor with disturbance observer and position estimator, In. ROBOTICS AND AUTONOMOUS SYSTEMS Volume: 79 Pages: 87-98 Published: MAY 2016.

[3] DUCHON, F. - HUBINSKY, P. - HANZEL, J.: et al.: Intelligent vehicles as the robotic applications, In: 5th International Conference on Modelling of Mechanical and Mechatronics Systems (MMaMS) Location: SLOVAKIA Date: NOV 06-08, 2012.

[4] Modelling of mechanical and mechatronics systems Book Series: Procedia Engineering Volume: 48 Pages: 105-114 Published: 2012.

[5] DUCHON, F. - HUNADY, D. - DEKAN, M.: et al.: Optimal navigation for mobile robot in known environment, In: 11th International Conference on Industrial, Service and Humanoid Robotics (ROBTEP 2012), ROBOTICS IN THEORY AND PRACTICE Book Series: Applied Mechanics and Materials Volume: 282 Pages: 33-+ Published: 2013.

[6] BABINEC, A. - DEKAN, M. - DUCHON, F.: et al.: Modifications of VFH navigation methods for mobile robots, In: 5th International Conference on Modelling of Mechanical and Mechatronics Systems (MMaMS), MODELLING OF MECHANICAL AND MECHATRONICS SYSTEMS Book Series: 
Procedia Engineering Volume: 48 Pages: 10-14 Published: 2012.

[7] VITKO, A. - JURISICA, L. - BABINEC, A.: et al.: Some didactic aspects of teaching robotics, In: 1st International Conference on Robotics in Education, PROCEEDINGS OF THE 1ST INTERNATIONAL CONFERENCE ON ROBOTICS IN EDUCATION Pages: 27-30 Published: 2010 .

[8] WebPage, 4.8.2017 : http://www.dobot.cc/

[9] BUSS, S. R.: Introduction to inverse kinematics with jacobian transpose, pseudoinverse and damped least squares methods. IEEE Journal of Robotics and Automation, 2004, 17.1-19: 16.

[10] BUSS, S. R.: 3-D Computer Graphics: A Mathematical Introduction with OpenGL, Cambridge University Press, 2003.

[11] JAYDEV P. DESAI: D-H Convention, Robot and Automation Handbook, CRC Press, 2005 USA, ISBN 0-8493-1804-1.

[12] ADELHARD BENI REHIARA: Kinematics of Adept Three Robot Arm, InTech, 2011, ISBN 978-953-307$160-2$

[13] KUCUK, S. - BINGUL, Z.: The Inverse Kinematics Solutions of Industrial Robot Manipulators, IEEE Conference on Mechatronics, pp. 274-279, Turkey, June 2004, Istanbul.

[14] KUCUK, S. - BINGUL, Z.: Robot Kinematics: Forward and Inverse Kinematics, in Industrial Robotics: Theory, Modelling and Control, 2007, ISBN 3-86611-285-8, p 128-148.

[15] KELEMEN, M. - FABIAN, M. - KELEMENOVA, T.: Design and Development of Lift Didactic Model Within Subjects of Mechatronics, In: 5th
International Conference on Modelling of Mechanical and Mechatronics Systems (MMaMS), MODELLING OF MECHANICAL AND MECHATRONICS SYSTEMS Book Series: Procedia Engineering Volume: 48 Pages: 280-286 Published: 2012.

[16] MIKOVA, L. - TREBUNA, F. - KELEMEN, M.: Concept of Locomotion Mobile Undercarriage Structure Control for the Path Tracking, In: MECHATRONIC SYSTEMS AND MATERIALS IV Book Series: Solid State Phenomena Volume: 198 Pages: 79-83 Published: 2013.

Received August 21, 2018, accepted November 13, 2019

\section{BIOGRAPHIES}

Ondrej Hock was born in 1985. In 2009 he graduated at the Department of mechatronics and electronics, Faculty of Electrical Engineering, University of Zilina. He defended his $\mathrm{PhD}$ in the field of programming device and systems in 2012; his thesis title was "Implementation of control algorithms with increased reliability in the FPGA programmable circuit “. Since 2012 he is working as a tutor with the Department of mechatronics and electronics. His scientific research is focusing on mechatronics.

Jozef Sedo was born in 1985 in Zilina, Slovakia. He graduated at the Faculty of Electrical Engineering, University of Zilina in 2011. He received his $\mathrm{PhD}$ degree in Power Electrical Engineering in 2016. He is currently working as an assistant professor in the Department of Mechatronics and Electronics at University of Zilina. His research interests include Mechatronics, Control Systems and Power Electronic. 